\title{
Proteomic analysis during of spore germination of Moniliophthora perniciosa, the causal agent of witches' broom disease in cacao
}

Joise Hander Mares ${ }^{1}$, Karina Peres Gramacho ${ }^{2}$, Everton Cruz Santos ${ }^{1}$, André da Silva Santiago ${ }^{3}$, Juliano Oliveira Santana ${ }^{1}$, Aurizângela Oliveira de Sousa ${ }^{1}$, Fátima Cerqueira Alvim ${ }^{1}$ and Carlos Priminho Pirovani ${ }^{*}$

\begin{abstract}
Background: Moniliophthora perniciosa is a phytopathogenic fungus responsible for witches' broom disease of cacao trees (Theobroma cacao L.). Understanding the molecular events during germination of the pathogen may enable the development of strategies for disease control in these economically important plants. In this study, we determined a comparative proteomic profile of $M$. perniciosa basidiospores during germination by two-dimensional SDS-PAGE and mass spectrometry.

Results: A total of 316 proteins were identified. Molecular changes during the development of the germinative tube were identified by a hierarchical clustering analysis based on the differential accumulation of proteins. Proteins associated with fungal filamentation, such as septin and kinesin, were detected only $4 \mathrm{~h}$ after germination (hag). A transcription factor related to biosynthesis of the secondary metabolite fumagillin, which can form hybrids with polyketides, was induced 2 hag, and polyketide synthase was observed 4 hag. The accumulation of ATP synthase, binding immunoglobulin protein (BiP), and catalase was validated by western blotting.

Conclusions: In this study, we showed variations in protein expression during the early germination stages of fungus M. perniciosa. Proteins associated with fungal filamentation, and consequently with virulence, were detected in basidiospores 4 hag., for example, septin and kinesin. We discuss these results and propose a model of the germination of fungus $M$. perniciosa. This research can help elucidate the mechanisms underlying basic processes of host invasion and to develop strategies for control of the disease.
\end{abstract}

Keywords: Basidiospore, Germination, Witches' broom, Proteomic analysis

\section{Background}

Moniliophthora perniciosa is a phytopathogenic fungus that belongs to class Basidiomycetes, order Agaricales, family Marasmiaceae [1]. This fungus causes witches' broom disease of cacao trees (Theobroma cacao L.) and caused the loss of $\sim 90 \%$ of cacao harvest in Central and South America in 1990 [2]. M. perniciosa is classified as hemibiotrophic, having two stages in its life cycle, the biotrophic (parasitic) phase with monokaryotic hyphae,

\footnotetext{
* Correspondence: pirovanicp@gmail.com

${ }^{1}$ Laboratory of Proteomics, Center of Biotechnology and Genetics, State

University of Santa Cruz (UESC), Ilhéus, Bahia, Brazil

Full list of author information is available at the end of the article
}

and the necrotrophic phase (saprophytic) with dikaryotic hyphae containing clamp connections [3]. Basidiospores are the infective propagules and can penetrate the host directly through the intact cuticle, natural openings of the cuticular surface, via epidermal cell junctions, at the base of trichomes, or through stomata $[4,5]$. The mycelium forms as hyphae grow within the plant, penetrating the plant tissue in direct contact with the host protoplasm; alternatively, mycelium can form on the plant surface [6]. Recent observations suggest that penetration by the basidiospore is related to the formation of a germination pore directly in the meristematic cuticle, followed by the emergence of a germinative tube [5]. 
During this infection phase, the fungus induces drastic physiological and morphological changes in the plant [7, 8]. After 2 to 3 months, the infected tissue enters a state of necrosis, and the affected branch of the plant dries and resembles a broom; this is the characteristic feature after which the disease is named [3, 9].

Accession and germination of fungal spores on the surface of plants are the initial steps essential for host penetration and colonization. Some studies have focused on molecular analysis of the germination of conidia and other types of phytopathogenic-fungi spores. Differentially expressed genes were identified in teliospores of Ustilago maydis, [10], in conidia of Fusarium graminearum [11], and in Botrytis cinerea [12]. In addition, analysis of protein profiles of filamentous-fungi spores identified proteins involved in the metabolism of carbohydrates, lipids, and proteins in Blumeria erysiphe [13]. Analysis of early stages of germination shows high expression of proteins associated with energy metabolism in conidia of both Aspergillus nidulans [14] and Colletotrichum acutatum [15]. Understanding the molecular events during germination may enable better strategies for disease control in these economically important plants. Nonetheless, few studies have been conducted to characterize and identify proteins involved in the germination of phytopathogenic fungi such as $M$. perniciosa.

The function of antioxidant proteins in spore survival has been barely reported. Nevertheless, some reports have revealed that enzymes involved in the stress response, e.g., superoxide dismutase (SOD) and catalase, show altered expression in Aspergillus niger conidiospores under temperature stress [16].

Modifications of expression patterns of heat shock proteins like binding immunoglobulin protein (BiP) may modulate the virulence of Pyrenophora tritici-repentis, the causal agent of tan spot of wheat [17]. A comparative analysis of proteins secreted by Verticillium dahlia during host invasion indicates that ATP levels change during infection [18]. Additionally, BiP seems to be involved in the virulence of other pathogens such as Bartonella henselae during host invasion [19].

Recently, our team developed methods for proteomic studies of $M$. perniciosa spores [20]. A protein extraction method and the quantity of basidiocarps and basidiospores required for proteomic studies were established, which allowed for the construction of a reference map based on two-dimensional (2D) polyacrylamide gel electrophoresis (PAGE). Next, a network of protein interactions was built through identification of proteins in nongerminated spores. In the present study, we determined the comparative proteomic profile of germinated and nongerminated basidiospores. In addition to the 175 protein spots identified by coincidence with the reference map for nongerminated-spore proteins [20], we identified
$>141$ unique protein spots seen 2 and $4 \mathrm{~h}$ after germination (hag). Molecular changes during the development of the germinative tube were identified by a hierarchical clustering analysis based on differential accumulation of proteins.

\section{Methods \\ Cultivation and collection of basidiospores}

Approximately 300 dry branches of a susceptible cacao cultivar (Catongo) between 30 and $50 \mathrm{~cm}$ long that were infected with the rosy mycelium of $M$. perniciosa [20] were randomly collected in the field (CEPLAC/CEPECIlhéus, Bahia) according to the methodology described by Mares and coworkers [20]. Basidiospores were produced by the method of Frias et al. [21] and stored in liquid nitrogen as described by Frias [22] until use.

\section{Germination of basidiospores and protein extraction}

Each aliquot of basidiospores was defrosted on ice, diluted in distilled water to reduce the concentration of glycerol to $3 \%$, and incubated in the dark at $25{ }^{\circ} \mathrm{C}$. Germination rates and lengths of the germinative tubes were monitored and measured with a graduated lens under a light microscope (Bioval L2000A) at 40× magnification.

The 0-, 2-, and 4-hag. samples were collected by centrifugation for $20 \mathrm{~min}$ at $5000 \times g$ in $3 \%$ glycerol. The supernatants were discarded, then the precipitates were snap-frozen in liquid nitrogen and stored at $-80^{\circ} \mathrm{C}$ until protein extraction. The samples stored at $-80^{\circ} \mathrm{C}$ were defrosted and washed with cold acetone containing $0.07 \% \mathrm{v} \cdot \mathrm{v}^{-1} \beta$-mercaptoethanol. Then, protein extraction and quantification were performed according to methods described by Mares and coworkers [20].

\section{D electrophoresis}

For proteomic analysis by 2D PAGE, $350 \mu \mathrm{g}$ of total protein diluted in $250 \mu \mathrm{L}$ of the rehydration solution (7 mol.L $\mathrm{L}^{-1}$ urea, 2 mol.L $\mathrm{L}^{-1}$ thiourea, 2\% CHAPS, and 0,002\% bromophenol blue) was used. Gel strips (containing immobiline) $13 \mathrm{~cm}$ long with an immobilized nonlinear $\mathrm{pH}$ gradient from 3 to 10 (Amersham Biosciences, Immobiline $^{\mathrm{TM}}$ Dry-Strip) were hydrated for $12 \mathrm{~h}$ in the rehydration buffer containing the total protein solution by the methods of Mares and coworkers [20] in an Ettan IPGphor 3 Isoelectric Focusing System. The second dimension was resolved in a $12.5 \%$ polyacrylamide gel in a HOEFER SE 600 Ruby vertical electrophoresis system (Amersham Bioscience). The run started with an electrical current of $15 \mathrm{~mA}$ per gel for $15 \mathrm{~min}$, followed by $30 \mu \mathrm{A}$ per gel for $30 \mathrm{~min}$, and $50 \mu \mathrm{A}$ per gel for $3.5 \mathrm{~h}$, totaling $4.25 \mathrm{~h}$. The gels were prepared in triplicate for each time stage hours after the start of germination. 


\section{Visualization of spots and image analysis}

After electrophoresis, the polyacrylamide gels were placed in fixation buffer (40\% ethanol and 10\% acetic acid) for $1 \mathrm{~h}$, then the buffer was replaced with colloidal Coomassie Blue dye $(8 \%$ ammonium sulfate, $0.8 \%$ phosphoric acid, 0.08\% Coomassie Brilliant Blue G-250, and $20 \%$ methanol), and the gels were incubated for 5 days with gentle rocking. The dye was replaced with distilled water, and the gentle rocking of the gels was continued with daily water changes until the excess dye was removed.

Gel images were scanned with a Labscanner (Amersham Bioscience) and analyzed for detection and relative quantification of protein spots using the ImageMaster 2D Platinum 7.0 software (GE Healthcare), considering the area and intensity of the spots. The reference gels (master gel) from triplicates were used to identify the unique spots and compare the relative accumulation of proteins present in the different treatment groups. The spots previously identified in the reference map [20] were compared with the spots on gels for 2 and 4 hag. As for the spots shared among different treatment groups, those that showed a relative fold change in abundance greater than 1.5 were designated as differentially expressed, and statistical significance was determined if $p<0.05$ in ANOVA. The gels from 2- and 4-h treatment groups were compared with the gels of nongerminated spores obtained by Mares and colleagues [20], which served as a reference (Additional files 1 and 2: Supplementaty Tables 1 and 2).

\section{Protein identification by mass spectrometry}

The spots differentially expressed between 2 and 4 hag. were excised from gels and placed in microtubes. Then, they were bleached and subjected to trypsin digestion [23]. Next, the samples containing recovered peptides were vacuum concentrated until they reached a volume of $10-15 \mu \mathrm{L}$.

The resulting peptides from tryptic digestion were subjected to liquid chromatography with tandem mass spectrometry (LC-MS/MS) on a nanoAcquity system (Waters, Milford, MA) coupled with a Q-ToF micro mass spectrometer (Waters), according to methods described elsewhere [23]. The raw data were processed, and the resulting spectra were analyzed in the ProteinLynx Global Server 4.2 software (Waters) and compared with the SwissProt database (http://www.uniprot.org/ downloads, October 2011). For comparison with the NCBI database, the MASCOT tool MS/MS IonSearch (www.matrixscience.com) was used with the following settings: tryptic digestion, with 1 cleavage site lost, cysteines modified by carbamidomethylation and methionine oxidation, error tolerance for the peptide of $30 \mathrm{ppm}$, and fragment mass error of MS/MS equal to 0.1 Da. According to MASCOT analysis probability, only the significant "hits" $(p<0.05)$ were accepted. After protein identification, their ontology and biological processes were classified in Blast2Go.

\section{Validation of proteomic analysis by western blotting} Aliquots of $10 \mu \mathrm{g}$ of total basidiospore protein $(0,2$, and 4 hag) were separated by $12.5 \%$ SDS-PAGE and transferred to a nitrocellulose membrane using the iBlot Dry Blotting System (Invitrogen). The membrane was blocked with 5\% skim milk $(w / v)$ in TBS-T buffer $\left(100 \mathrm{mmol} \cdot \mathrm{L}^{-1} \mathrm{Tris}-\mathrm{HCl}\right.$, $\mathrm{pH} 8.0 ; 140 \mathrm{mmol} \cdot \mathrm{L}^{-1} \mathrm{NaCl} ; 0.05 \% \mathrm{v} \cdot \mathrm{v}^{-1}$ Tween 20$)$. BiP $(71 \mathrm{kDa})$, catalase $(83 \mathrm{kDa})$, and ATP synthase $(53 \mathrm{kDa})$ were detected using polyclonal primary antibodies (at 1:2000 dilution) against the following proteins: BiP of Arabidopsis thaliana (Agrisera-AS09481), catalase of $A$. thaliana (501,100 Agrisera-AS09), and ATP synthase of $A$. thaliana (Agrisera-AS05085). The membranes were incubated with the appropriate primary antibody for $60 \mathrm{~min}$. After three washes with TBS-T buffer, the membranes were incubated for $60 \mathrm{~min}$ with a secondary antibody: a goat anti-rabbit IgG antibody conjugated with alkaline phosphatase (Thermo Fisher Scientific-65-6122). The phosphate of 5-bromo-4-chloro-3-indolyl (BCIP) and p-nitrotetrazolium (NBT; Promega, USA) served as substrates for the colorimetric reaction of alkaline phosphatase. The accumulation of proteins BiP, catalase, and ATP synthase was quantified by means of the membrane images using the GelQuant.Net 1.8.0 software (www.biochemlabsolutions.com). To confirm equal distribution of proteins, the membranes were washed with $0.1 \mathrm{M}$ glycine, $\mathrm{pH} 2.9$, for western blotting membrane stripping, and a new labeling was made. The revelation time was increased until background bands shows up to indicates the homogeneous transfer among the samples.

\section{Hierarchical clustering analysis}

Clustering was performed using the Cluster $3.0+$ Java TreeView software (http://bonsai.hgc.jp/ mdehoon/software/cluster/software.htm). For this purpose, the matrix was built from normalized log-transformed ratios for each protein spot from the analysis of gel images in the Image Master 2D Platinum 7.0 software (GE Healthcare); (Additional file 1: Table S1). Euclidean distance (ED) was used to calculate the distance or dissimilarity between individuals and Complete Link was used for clustering.

\section{Results and discussion}

\section{The protein profile according to 2D SDS-PAGE}

Previously, we established a reference map for proteins in nongerminated $M$. perniciosa spores [20]. In the present study, we determined the protein profiles of $M$. perniciosa spores 2 and 4 hag. and compared them with the previously generated data. These collect points were demonstrated as being the necessary time to complete 
translocation of the intracellular content from the spore to germinative pore. This was noted due to the lack of staining of the basidiospore 4 after the germination, which is considered complete germination [20].

A total of 141 proteins were identified by mass spectrometry, totaling 319 when combined with those identified by Mares and colleagues [20]. The number of proteins identified in other proteomic studies included 118 in Botrytis cinerea spores [24], 130 in Rhizoctonia solani AG-1 sclerotia maturation $[25,26]$, and 365 in Colletotrichum acutatum conidia germination [15]. Thus, the present study identified one of the largest numbers of proteins in spores of a phytopathogenic fungus. In addition, the accumulation of proteins BiP, catalase A, and ATP synthase $\beta$ subunit was validated by western blotting with antibodies against the A. thaliana proteins. The homology of these $M$. perniciosa proteins with the corresponding A. thaliana proteins is 66,48 , and $66 \%$, respectively. Alignments between the proteins of these two species showed blocks of conserved amino acid residues long enough for the presence of reactive epitopes that can be detected by polyclonal antibodies (Additional file 2: Figure S1). Next, we will discuss the comparative proteomic profiles corresponding to the germination of M. perniciosa basidiospores.
2D gels of basidiospore proteins for germination time points 0,2 , and $4 \mathrm{~h}$ were analyzed for variation in abundance and distribution of spots to obtain protein profiles (Fig. 1a). Proteins were visualized in the whole $\mathrm{pH}$ range, and molecular weight (MW) was noted. A greater abundance of proteins with MW above $90 \mathrm{kDa}$ is seen in the 2and 4-hag. samples as compared to baseline (Fig. 1a). The gel analysis revealed 510, 430, and 504 spots for the germination time points 0,2 , and $4 \mathrm{~h}$, respectively. A total of 242 spots were common among all the three treatment groups (Fig. 1b). Thirty percent (153 spots) of spots identified in nongerminated basidiospores were unique to this group. Basidiospores at 2 hag. had 26\% (112) unique spots. Basidiospores at 4 hag. had 37\% (187) unique spots.

All the treatment groups were evaluated concerning the distribution of spots relative to their MW and isoelectric point (pI; Additional file 3: Figure S2 and Additional file 4: Figure S3). Most proteins that constitute the proteomic map for the three time points under study are distributed in the range of 30 to $60 \mathrm{kDa}(63.4,64.8$, and $53.5 \%$ for 0 , 2 , and $4 \mathrm{~h}$, respectively). A $31.5 \%$ increase was observed in the percentage of proteins with MW greater than $60 \mathrm{kDa}$ in the spores 4 hag. The percentage of spots with MW less than $30 \mathrm{kDa}$ increased by $\sim 6 \%$ from 0 to 2 hag. and decreased by $10 \%$ from 2 to 4 hag. (Additional file 2 :

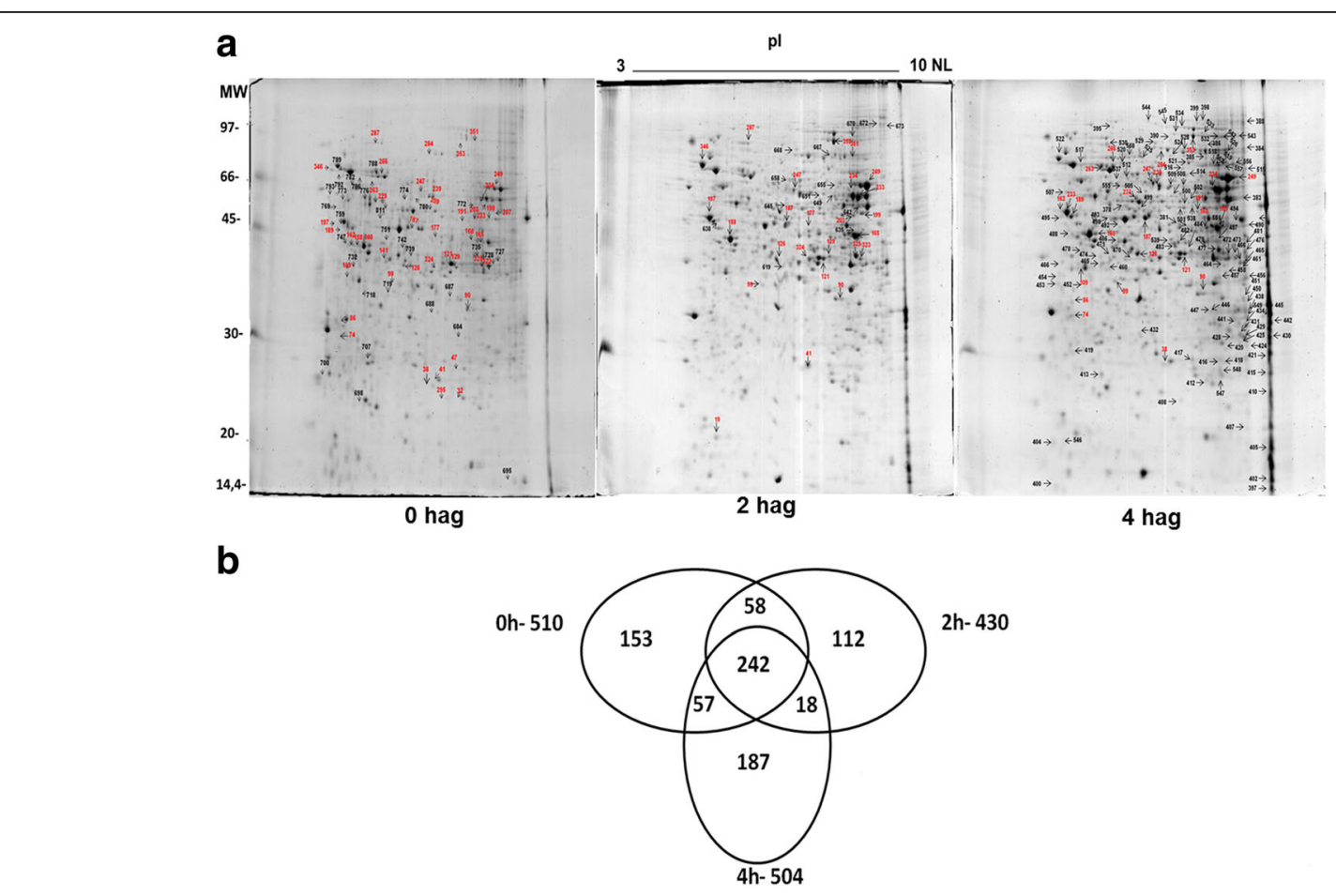

Fig. 1 Protein profile in 2DE-PAGE at different stages of $M$. perniciosa spore germination. a Non-germinated basidiospores of $M$. perniciosa (0 h) (Mares, et al., [20]) with 2 and $4 \mathrm{~h}$ after germination. The samples were focused in strips of $13 \mathrm{~cm}$ with nonlinear (NL) pH gradient 3-10. Black numbers correspond to unique spots for each treatment. Red numbers correspond to spots common between treatments with fold change $>1.5$. b Venn diagram of the distribution of spots detected in gels of 0 (Mares et al., [20]), 2, and $4 \mathrm{~h}$ after basidiospore germination 
Figure S1). The images show predominance of acidic proteins (isoelectric points less than 5.5) in nongerminated basidiospores compared to germinated spores. On the other hand, the number of basic proteins (isoelectric points greater than 7.5) in nongerminated spores was generally less than the number of basic proteins detected in germinated spores. Although the gels for 2-h and 4-h samples do not have spots in all $\mathrm{pH}$ ranges seen on the 0-h gels, the distribution of proteins on the basis of their pI was homogeneous in the ranges that contained spots (Additional file 3: Figure S2).

A total of 109 spots common for all three treatment groups, as identified by MS/MS, were analyzed and clustered according to their profile. Proteins that showed similar accumulation patterns were grouped by hierarchical clustering (Fig. 2). Group A contains proteins that showed reduced expression with time after germination. Group B contains proteins that showed an increase in expression 2 hag. but then were downregulated at 4 hag. Group $\mathrm{C}$ contains proteins that showed only accumulation during germination. More information on the identification of spots can be found in Additional file 1: Table S1.

\section{Most spots with high molecular weight correspond to enzymes associated with metabolic processes}

Unique and differentially expressed proteins were classified into categories by their biological processes as determined by the Blast2GO software. Some categories were chosen to be discussed because of their differential accumulation between 2 and 4 hag, relative to nongerminated spores as a reference [20]. The categories chosen were oxidation-reduction, development, biosynthetic, and metabolic processes, the stress response, and regulation. When compared with nongerminated spores, $40 \%$ (6) and $46.1 \%$ (6) of proteins related to oxidation-reduction were downregulated in treatment groups 2 and 4 hag., respectively. Two proteins related to fungal development showed accumulation at 2 hag., and one was upregulated 4 hag. Among all the proteins detected in nongerminated spores, 15 were classified into the category related to biogenesis and biosynthetic processes yet none of these proteins appeared at 2 hag. Four hag., 79.2\% of biosynthetic proteins showed reduced accumulation, and 20.8\% showed increased accumulation when compared with nongerminated spores. The category related to metabolic processes was the most represented, with $40 \%$ of the proteins showing reduced accumulation and $60 \%$ showing increased accumulation 2 hag., and at 4 hag., $52.7 \%$ of proteins showed reduced accumulation and 51.3\% showed increased accumulation. Among the proteins involved in stress response, $80 \%$ of proteins showed reduced accumulation and $20 \%$ showed hyperaccumulation at 2 and 4 hag., respectively. At 2 hag., $62.5 \%$ of the proteins involved in regulation showed increased accumulation, and at 4 hag., $60 \%$ of the proteins showed decreased accumulation (Fig. 3).

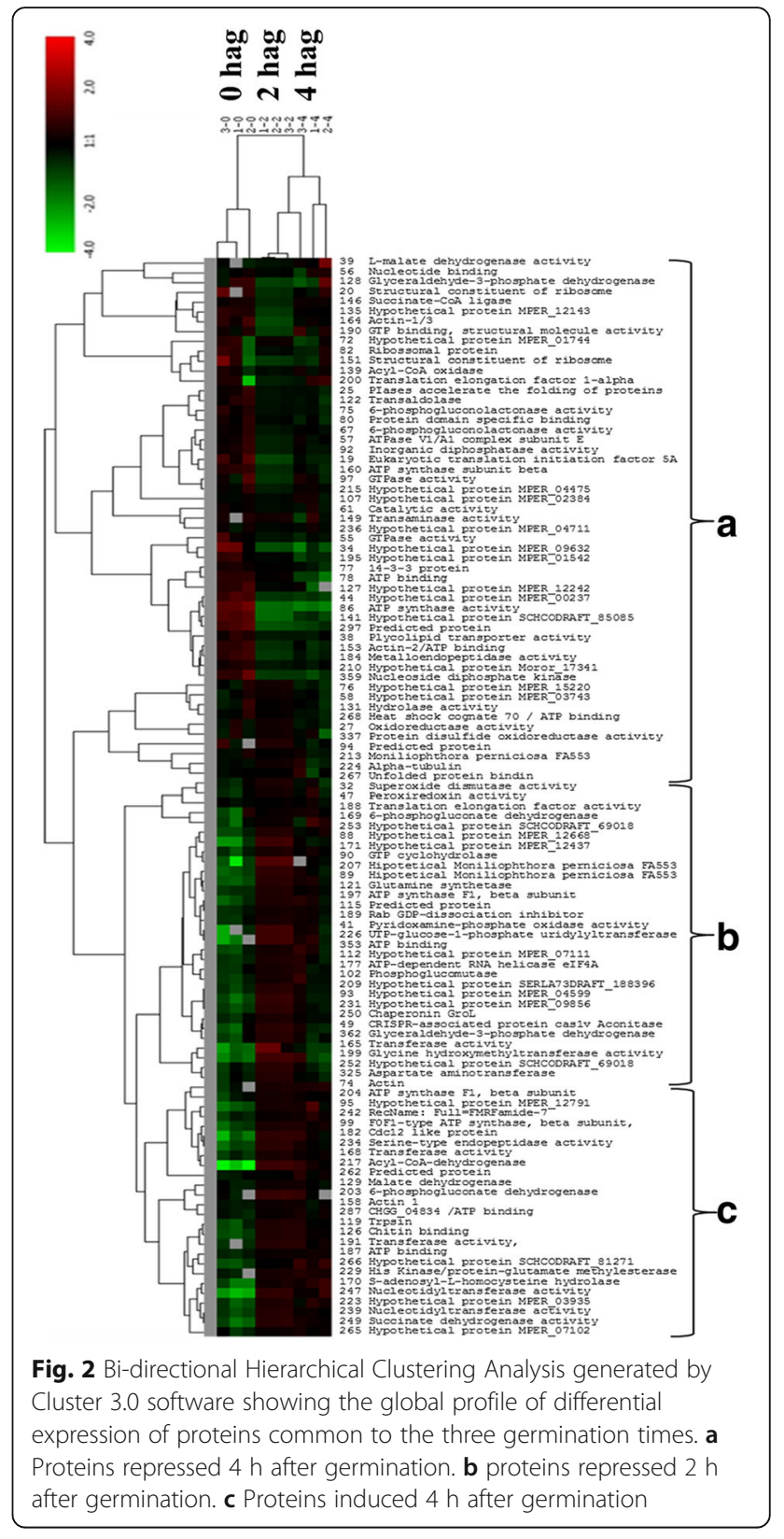

The increase in the amount of high-molecular-weight proteins in spores 4 hag may be related to the increased expression of energy production machinery. Most high-molecular-weight spots correspond to enzymes associated with metabolic processes, for example, ATP synthase $(68.1 \mathrm{kDa})$, ATP-binding protein $(68.8 \mathrm{kDa})$, and succinate dehydrogenase $(62.3 \mathrm{kDa})$.

The distribution of spots in gels is a qualitative result that facilitates the choice of $\mathrm{pH}$ and $\mathrm{MW}$ range in the subsequent studies. Twenty acidic proteins were identified by mass spectrometry. Acidic-protein enrichment techniques have been tested on the samples in 2D electrophoresis to analyze differences in the accumulation 

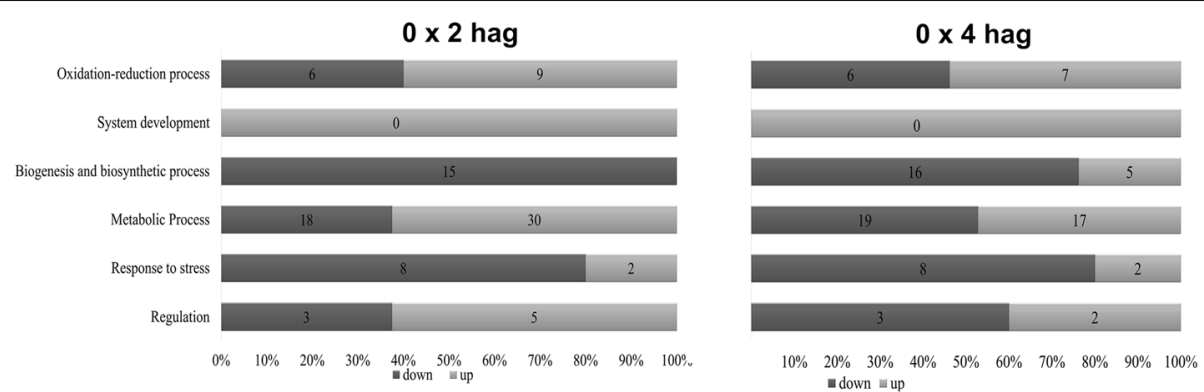

Fig. 3 Classification of biological processes for differentially expressed proteins identified in non-germinated spores of M. perniciosa, compared with 2 and $4 \mathrm{~h}$ after germination. Classification was performed using Blast2GO software

between acidic subproteomes of yeast and hyphae in Candida albicans [27]. This analysis can also be conducted to identify proteins in acidic subproteomes of phytopathogenic fungi such as $M$. perniciosa and M. roreri. The acidic proteins identified in the present study participate in regulation of important molecular functions (spots 15, 36, 77, 80, and 88), cytoskeleton organization (spots 97 and 305), energy metabolism (spots 19, 160, 184, 195, 304, and 700), and a stress response (spots 270, 337, 714, 751, and 811). Among these proteins, the only ones related to energy metabolism that were downregulated 2 and 4 hag. were translation initial factor $C$ (spot 19) and ATP synthase $\beta$ subunit (spot 160), respectively.

\section{Proteins related to metabolism and energy}

Proteins associated with protein metabolism showed progressively decreasing abundance throughout germination: translation initial factor (spot 19) and nucleoside diphosphate kinase (spot 297) decreased at 2 hag. (Additional file 5: Table S2), and small ribosomal subunit (spot 109) and heat shock protein (spot 263) decreased 4 hag. (Additional file 5: Table S2). Two other proteins showed reduced expression after the onset of germination (spots 34 and 44; Fig. 2a). Although these proteins were not characterized in the $M$. perniciosa database, BLASTP analysis showed that these spots correspond to proteins participating in the metabolism of proteins, e.g., 605 ribosomal protein (spot 34) and serine protease inhibitor (spot 44). This finding may be related to the stress response and synthesis of new proteins when a basidiospore prepares for the growth of the germinative tube [20]. In addition, several heat shock proteins and other stress-related proteins have been demonstrated to improve the ability of nongerminated conidia to survive various types of environmental stress [28].

Other proteins related to protein metabolism were induced, and some were exclusively detected at 4 hag. (Fig. 3). These proteins include nucleoside triphosphate hydrolase proteins (spot 384), constituents of larger subunits of ribosomes (spot 397), translation elongation factor $1 \alpha$ (spot 383), folding protein (spot 390), and HSP70 (spot 522; Additional file 1: Table S1). Compared to nongerminated basidiospores, BiP showed upregulation of $\sim 2.8$-fold at 2 hag. and $\sim 3.4$-fold at 4 hag. BiP upregulation correspond to the expression of a few spots related to HSP70 (spots 268 and 278) that were common among the treatments. As expected, the final germination phase of $M$. perniciosa basidiospores requires expression of some of these proteins for transcription and synthesis of new proteins. In addition, upregulation of $\mathrm{BiP}(\mathrm{a}$ molecular chaperone from the HSP70 family), i.e., Spot 278, resident in the endoplasmic reticulum (ER), occurred 4 hag. as shown by the cross-reaction with the antibody against $\mathrm{BiP}$ of $A$. thaliana (Fig. 4a and b). BiP also plays a central role as a sensor of environmental stressors in the ER, affecting the assembly and folding of proteins $[29,30]$. BiP induction during the germination of $M$. perniciosa is suggestive of an increase in ER secretory activity [31]. This finding is in agreement with proteomic studies on $A$. nidulans germination, where multiple heat shock proteins were found to be upregulated 30 and 60 min after the onset of germination [14].

Proteins with increased expression during germination were clustered (Fig. 2b). Among these, six are members of the ATP synthase family (spots 204, 95, 242, 99, 187, and 266). Accumulation of ATP synthase can be noted in western blot analysis (spots 197 and 204) performed in this study. These proteins may be related to a direct increase in metabolic activity and energy production needed to meet the energy demand for the development of the germinative tube. Conversely, glyceraldehyde-3phosphate dehydrogenase (spot 128, Fig. 2a) was downregulated after the onset of germination. This phenomenon may be related to the temporary accumulation of glycerol during germination. The same phenomenon has been observed in Phycomyces blakesleeanus and seems to be caused by the activation of cAMP-dependent glycerol-3-phosphatase: an enzyme that indirectly participates in the glycolytic pathway [32] Excessive increase of glycerol production during spore germination can cause a buildup intracellular osmotic pressure [33]. This observation may explain the swelling 

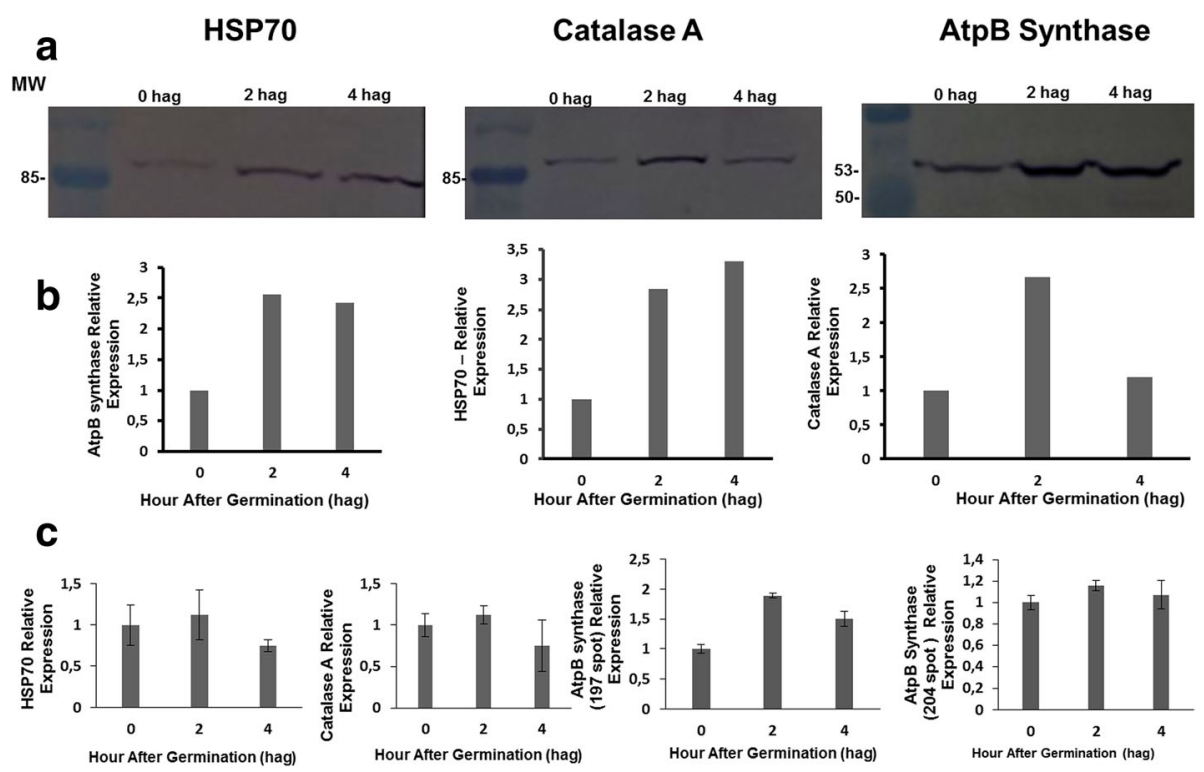

Fig. 4 Accumulation of BiP, Catalase, and ATP synthase (beta chain) by western blot and analysis of spots. a Image of a nitrocellulose membrane hybridized with Anti-BiP, Anti-Catalase, and Anti-ATP synthase (beta chain) of Arabidopsis (Normalization, see Additional file 6: Figure S4). b Relative accumulation determined from images of the nitrocellulose membrane, using the Gel Quant. Net 1.8.0 software. c Relative quantification of each spot corresponding to proteins analyzed by western blot. The average values of the each spot's percentage volume in each gel replicate was used for graph construction

observed in $M$. perniciosa basidiospores before the start of the germinative tube emission, i.e., from 0 to $30 \mathrm{~min}$ after defrosting and introduction of basidiospores [20].

Cell cycle-related proteins and septation of the primary hypha Valosin-containing protein (VCP) was identified (spot 304), which is a member of the AAA ATPase family (ATPases associated with a variety of activities) and contains two conserved domains also called AAA [34]. VCP is a homolog of CDC48p, which is well conserved among all eukaryotes and is essential for growth of Saccharomyces cerevisiae [7]. This protein performs a critical function in regulation of the cell cycle, and is required for entry into mitosis [35]. During the germination of basidiospores, this protein can be associated with the process of cell division and consequently with the growth of hyphae. Similarly, a kinesin (spot 395) was identified, which is a motor protein with an important role in cell division. Kinesins are necessary for proper length and sliding of microtubules inside the spindle during prophase and metaphase and for microtubule depolymerization during anaphase [36].

Septin (spot 488) is another protein found only in spores 4 hag. Since its discovery in S. cerevisiae [37], other septins have been found in eukaryotes, especially in filamentous fungi, where septins control the filamentous morphology. During septum formation, the septin ring splits in two to form a double ring. Due to their lack of septa, septin mutants are highly sensitive, and damage to a single hypha can result in complete lysis of a young mycelium [38]. In studies conducted on phytopathogenic basidiomycete $U$. maydis, gene Sep3 was found to perform important functions in morphogenesis of the filamentous cells, in the corn infection process, and in symptom development [39]. In Magnaporthe ory$z a e$, septins mediate cortex rigidity and curvature of the membrane necessary for spore penetration of a rigid plant part, like the cuticle of a leaf, to facilitate infection [40]. To analyze septin function, some targeted drugs can be used; however, for the most part, these compounds are not readily available. One such compound is forchlorfenuron (FCF; N-[2-Chloro-4-pyridyl]-N'-phenylurea; HPM300), a plant cytokinin able to quickly induce production of abnormal septin structures in S. cerevisiae [41]. To gain an insight into how FCF binds to septin proteins, simulations were carried out in silico on the basis of docking of FCF's structure to all the highresolution crystal structures of septin available. These studies suggested that FCF mimics nucleotides and thus interferes with the dynamics of GTP-binding proteins by modifying the mounting base of septin filaments [42]. In this regard, we recommend that the septin protein be used in studies on effective control of the invasion of young tissues of cacao plants by fungus $M$. perniciosa.

\section{The response to stress-related proteins}

Proteins active in the stress response are present among the ones detected exclusively in nongerminated basidiospores. Oxidoreductases (spots 684 and 687) and ascorbate peroxidase (spot 688) belong to this category (Fig. 3). 
These proteins may be involved in a fungal adaptation intended to neutralize reactive oxygen species (ROS) produced by plants as a defense mechanism [23]. In addition, other studies have indicated that oxidative respiration is absolutely required for germination of fungal spores [43, 44].

The relative expression of catalase A detected in 2D gels of proteins at 0,2 , and 4 hag. correlates with the expression of this protein detected by western blotting with an anti-CatA antibody specific for the A. thaliana protein (Fig. $4 \mathrm{a}-\mathrm{C}$ ). An increase in catalase expression can be observed 2 hag. This change may contribute to the survival of basidiospores and mycelium of the biotrophic type of $M$. perniciosa treated with $4 \mathrm{mM}$ hydrogen peroxide in vitro [45]. In addition, there was upregulation of SOD (spot 32) at 2 hag. (Table 1). SOD expression, combined with the accumulation of peroxiredoxin (spot 47; Fig. 2b) and catalase, points to the need for neutralization of free radicals within the germinative tube during germination. Similarly, accumulation of catalase A during the germinative process of $A$. nidulans in the first $30 \mathrm{~min}$ after germination has been observed elsewhere [14].

\section{Fungal-virulence-related proteins}

Peptidyl-prolyl cis-trans isomerase (spot 695) is a relevant protein that was detected exclusively in nongerminated spores (Additional file 1: Table S1). This protein is associated with the pathogenicity of $B$. cinerea, being characterized as a virulence factor important for host invasion [46]. These data suggest that nongerminated basidiospores of M. perniciosa accumulate virulence factors essential for a quick attack on the host in a manner similar to the mode of action of B. cinerea conidia [23].

Our results suggest that a secondary terpenoid metabolite synthesis route associated with polyketides can be enabled in M. perniciosa during germination [47]. Transcription factor FapR (Table 1, spot 323), which regulates both clusters of genes involved in the biosynthesis of secondary metabolites fumagillin and pseurotin in Aspergillus fumigatus [47], was induced 2 hag. The metabolite fumagillin is a hybrid polyketide-terpenoid compound [48]. Additionally, polyketide synthase (gi|392,596,225) was identified exclusively 4 hag. (Table 2 , spot 388 ). Polyketide synthase belongs to a large family of enzymes responsible for production of various secondary metabolites such as pigments, toxins, antibiotics, and signaling molecules [49]. This protein catalyzes the first reaction of melanin biosynthesis [50] and may participate in the synthesis of fumagillin [47]. Melanin production by pathogenic fungi can contribute to their virulence toward humans and cultivated plants [51].

\section{Conclusion}

Generally, the comparative proteomic analysis based on a combination of 2D-SDS-PAGE with MS/MS enabled identification of a general scheme of biological processes that take place during the initial developmental phase of fungus M. perniciosa (Fig. 5).

In conclusion, in this study, we showed variations in protein expression at the early germination stages of $M$. perniciosa. Some proteins related to metabolism are expressed as the germinative tube develops. Protein synthesis intensifies during germination, judging by the accumulation of ribosomal components, elongation factors, and BiP. Upregulation of oxidative-stress-related proteins such as SOD and catalase can facilitate detoxification of free radicals inside the primary hypha. Proteins associated with fungal filamentation, and consequently with virulence, were detected in basidiospores 4 hag., for instance, septin and kinesin. This finding indicates that the germinative tube is preparing for primary hypha

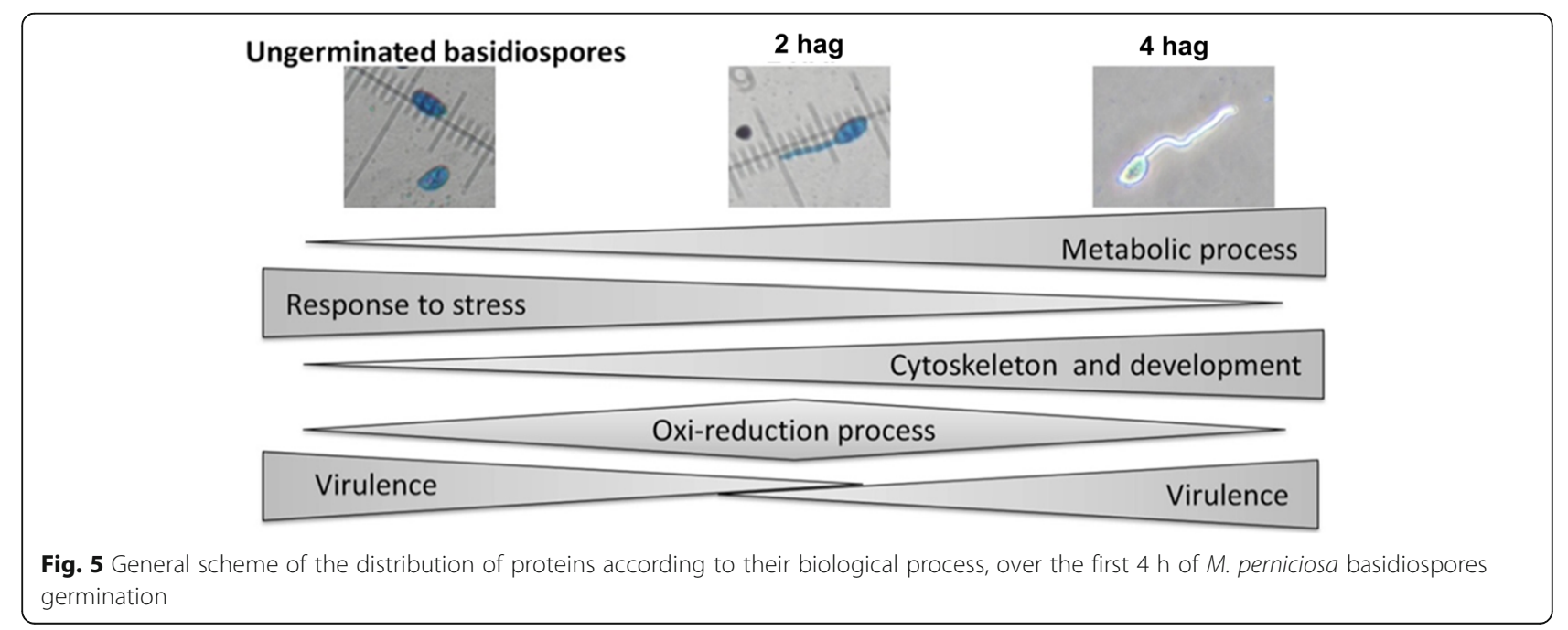


differentiation and host invasion. These proteins are promising targets for strategies of fungal control, when inhibitory compounds become available. Additionally, it is possible that pathogenicity-related proteins are specific to each step of the process of fungal infection. Virulence factors such as polyketide synthase and FapR are present in basidiospores and the primary hypha. Accordingly, we propose a model of the germination of fungus $M$. perniciosa (Fig. 5). This research may help to unravel the mechanisms behind the basic processes that this pathogen evolved to survive in (and to invade) the host tissue, causing one of the diseases responsible for huge losses in cacao cultivation in South and Central America.

\section{Additional files}

Additional file 1: Table S1. Ratio values for each protein spot used to perform the hierarchical clustering analysis. (XLS $61 \mathrm{~kb}$ )

Additional file 2: Figure S1. Sequence alignment of the target proteins identified in the western blots and their homologous in plant recognized by the antibodies. A - Sequence alignment between BiP from A. thaliana and the HSP70 from Moniliophthora perniciosa. B Sequence alignment of the ATP synthase from A. thaliana and its homologous in Moniliophthora perniciosa. C - Sequence alignment of the catalase A from A. thaliana and its homologous in Moniliophthora perniciosa. (JPEG $3987 \mathrm{~kb}$ )

Additional file 3: Figure S2. Distribution of the spots and their respective molecular weight in the 0,2 and 4 hag. (TIFF $2477 \mathrm{~kb}$ )

Additional file 4: Figure S3. Distribution of the spots and their respective isoelectric point in the 0,2 and 4 hag. (TIFF $741 \mathrm{~kb}$ )

Additional file 5: Table S2. Total spots identified in the MS/MS. In sequence, mw corresponds to a molecular mass values, the isoelectric point pl estimated; number of peptides by MS / MS; Score of sequence, sequence coverage and peptide sequence. All values were calculated by Mascot at http:. //www.matrixscience.com. (XLS $118 \mathrm{~kb})$

Additional file 6: Figure S4. Background detection by western blotting by overincubating the membranes. The anbidodies against Catalase and HSP70 were very specific. However, the antibody against Catalase has revealed background bands that were homogeneously transferred to the membrane at 0, 2 and 4 hag. (TIFF $498 \mathrm{~kb}$ )

\section{Abbreviations}

2D: Two-dimensional; BiP: Binding immunoglobulin protein; hag: Hours after germination; LC-MS/MS: Liquid chromatography with tandem mass spectrometry; PAGE: Polyacrylamide gel electrophoresis; SOD: Superoxide dismutase

\section{Acknowledgments}

This work was supported by grants from FAPESB (Fundação de Apoio à Pesquisa do Estado da Bahia) and CNPq (Conselho Nacional de Desenvolvimento Científico e Tecnológico). We would like to express our gratitude to the phytopathology laboratory staff at CEPEC/CEPLAC for support with the collection of basidiospores and to Louise Araujo Brito and Renata for assistance with basidiospore collection. C. P. Pirovani was supported by research fellowships from CNPq (Pq 1D - 304536/2015-6).

\section{Funding}

This work was supported by Brazilian funding agencies Conselho Nacional de Desenvolvimento Científico e Tecnológico (Pq 1D - 304,536/2015-6) and Fundação de Amparo à Pesquisa do Estado da Bahia (PRONEM PNE 005/ 2011). Funding body not participated in the design of the study and collection, analysis, and interpretation of data and in writing the manuscript.

\section{Availability of data and materials}

All data generated or analysed during this study are included in this published article and its supplementary information files, except the $\mathrm{ms} / \mathrm{ms}$ spectra that are available with correspondent author of this manuscript.

\section{Authors' contributions}

Conception and design of the work: JHM, KPG, and CPP. Obtaining of the data: JHM, ECS, ASS, and AOS. Analysis and interpretation of the data: JHM, $K P G, E C S, A S S, A O S, F C A$, and CPP. Drafting the manuscript or revising it critically for important intellectual content: JHM, KPG, ECS, ASS, AOS, FCA, and CPP. Final approval of the version to be published: JHM, KPG, ECS, ASS, AOS, FCA, CPP. Agreement to be accountable for all aspects of the work: JHM and CPP. All authors read and approved the final manuscript.

Ethics approval and consent to participate

This work did not need formal ethics approval according to 466/2012 Resolution of the CNS and Brazilian guideline for the care and use of animals in teaching or scientific research activities (DBCA Lay $N^{\circ} 11,794 / 2008$ ).

\section{Consent for publication}

No applicable.

\section{Competing interests}

The authors declare that they have no competing interests.

\section{Publisher's Note}

Springer Nature remains neutral with regard to jurisdictional claims in published maps and institutional affiliations.

\section{Author details}

${ }^{1}$ Laboratory of Proteomics, Center of Biotechnology and Genetics, State University of Santa Cruz (UESC), Ilhéus, Bahia, Brazil. '2Laboratory of Plant Pathology, Cacao Research Center, CEPLAC, Ilhéus, Bahia, Brazil. ${ }^{3}$ Center for Molecular Biology and Genetic Engineering (CBMEG) Unicamp, São Paulo, Brazil.

Received: 29 September 2016 Accepted: 9 August 2017

Published online: 17 August 2017

\section{References}

1. Aime MC, Phillips-Mora W. The causal agents of witches' broom and frosty pod rot of cacao (chocolate, Theobroma cacao) form a new lineage of Marasmiaceae. Mycologia. 2005;97:1012-22.

2. Purdy LH, Schmidt RA. Status of cacao witches' broom: biology, epidemiology, and management. Annu Rev Phytopathol. 1996;34:573-94.

3. Evans HC. Pleomorphism in Crinipellis perniciosa, causal agent of witches' broom disease of cocoa. Trans Br Mycol Soc. 1980;74:515-23.

4. Kilaru A, Hasenstein KH. Development and pathogenicity of the fungus Crinipellis perniciosa on interaction with cacao leaves. Phytopathology. 2005:95:101-7.

5. Sena K, Alemanno L, Gramacho KP. The infection process of Moniliophthora perniciosa in cacao. Plant Pathol. 2014;63:1272-81.

6. Anderbrhan T. Studies on the epidemiology and control of witches' broom disease of cocoa in the Brazilian Amazon. In: 9th International Cocoa Research Conference: 1984. p. 8.

7. Meinhardt LW, et al. Moniliophthora perniciosa, the causal agent of witches' broom disease of cacao: what's new from this old foe? Mol Plant Pathol. 2008:9:577-88.

8. Scarpari LM, et al. Biochemical changes during the development of witches' broom: the most important disease of cocoa in Brazil caused by Crinipellis perniciosa. J Exp Bot. 2005;56:865-77.

9. de Oliveira Ceita G, et al. Involvement of calcium oxalate degradation during programmed cell death in Theobroma cacao tissues triggered by the hemibiotrophic fungus Moniliophthora perniciosa. Plant Sci. 2007;173:106-17.

10. Zahiri AR, Babu MR, Saville BJ. Differential gene expression during teliospore germination in Ustilago maydis. Mol Gen Genomics. 2005;273:394-403.

11. Seong KY, et al. Conidial germination in the filamentous fungus Fusarium graminearum. Fungal Genet Biol. 2008;45:389-99.

12. Doehlemann G, Berndt P, Hahn M. Different signalling pathways involving a Galpha protein, CAMP and a MAP kinase control germination of Botrytis cinerea conidia. Mol Microbiol. 2006;59:821-35. 
13. Noir $\mathrm{S}$, et al. A proteomic analysis of powdery mildew (Blumeria graminis $\mathrm{f}$ sp. hordei) conidiospores. Mol Plant Pathol. 2009;10:223-36.

14. Oh YT, et al. Proteomic analysis of early phase of conidia germination in Aspergillus nidulans. Fungal Genet Biol. 2010;47:246-53.

15. El-Akhal MR, et al. Proteomic analysis of conidia germination in Colletotrichum acutatum. Arch Microbiol. 2013;195:227-46.

16. Cao T, Kim YM, Kav NNV, Strelkov SE. A proteomic evaluation of Pyrenophora tritici-repentis, causal agent of tan spot of wheat, reveals major differences between virulent and avirulent isolates. Proteomics. 2009;9(5):1177-96.

17. Abrashev R, Dolashka P, Christova R, Stefanova L, Angelova M. Role of antioxidant enzymes in survival of conidiospores of Aspergillus niger 26 under conditions of temperature stress. J Appl Microbiol. 2005;99(4):902-9.

18. Chu J, et al. Comparative analyses of secreted proteins from the phytopathogenic fungus Verticillium dahliae in response to nitrogen starvation. Biochim Biophys Acta. 2015;1854:437-48.

19. Chang CC, et al. A comparative study of the interaction of Bartonella henselae strains with human endothelial cells. Vet Microbiol. 2011;149:147-56.

20. Mares $\mathrm{JH}$, et al. Protein profile and protein interaction network of Moniliophthora perniciosa basidiospores. BMC Microbiol. 2016;16(1):120.

21. Frias GA, Purdy LH, Schimidt RA. Infection biology of Crinipellis perniciosa on vegetative flushes of cacao. Plant Dis. 1991;75:6.

22. Frias GA, Purdy LH, Schimidt RA. An inoculation method for evaluating resistance of cacao to Crinipellis perniciosa. Plant Dis. 1995;79:5.

23. Villela-Dias C, et al. Nep1-like protein from Moniliophthora perniciosa induces a rapid proteome and metabolome reprogramming in cells of Nicotiana benthamiana. Physiol Plant. 2014;150:1-17.

24. Gonzalez-Rodriguez VE, et al. Proteomic profiling of Botrytis cinerea conidial germination. Arch Microbiol. 2015;197:117-33.

25. Kwon YS, et al. Proteomic analysis of Rhizoctonia solani AG-1 sclerotia maturation. Fungal Biol. 2014;118:433-43.

26. Monteoliva $\mathrm{L}$, et al. Quantitative proteome and acidic subproteome profiling of Candida albicans yeast-to-hypha transition. J Proteome Res. 2011:10:502-17.

27. Cooper B, Garrett WM, Campbell KB. Shotgun identification of proteins from uredospores of the bean rust Uromyces appendiculatus. Proteomics. 2006;6:2477-84.

28. Hendershot LM. The ER function BiP is a master regulator of ER function. Mt Sinai J Med New York. 2004;71:289-97.

29. Malhotra JD, Kaufman RJ. The endoplasmic reticulum and the unfolded protein response. Semin Cell Dev Biol. 2007;18:716-31.

30. Reis PA, et al. The binding protein BiP attenuates stress-induced cell death in soybean via modulation of the $\mathrm{N}$-rich protein-mediated signaling pathway. Plant Physiol. 2011;157:1853-65.

31. van Schaftingen E, van Laere AJ. Glycerol formation after the breaking of dormancy of Phycomyces blakesleeanus spores. Role of an interconvertible glycerol-3-phosphatase. Eur J Biochem FEBS. 1985;148:399-404.

32. d'Enfert C. Fungal spore germination: insights from the molecular genetics of Aspergillus nidulans and Neurospora crassa. Fungal Genet Biol. 1997;21:163-72

33. Neuwald AF, et al. AAAt: A class of chaperone-like ATPases associated with the assembly, operation, and disassembly of protein complexes. Genome Res. 1999;9:27-43.

34. Frohlich $\mathrm{KU}$, et al. Yeast cell cycle protein CDC48p shows full-length homology to the mammalian protein VCP and is a member of a protein family involved in secretion, peroxisome formation, and gene expression. $J$ Cell Biol. 1991;114:443-53.

35. Fu X, Ng C, Feng D, Liang C. Cdc48p is required for the cell cycle commitment point at Start via degradation of the G1-CDK inhibitor Far1p. J Cell Biol. 2003;163:21-6.

36. Goshima G, Vale RD. Cell cycle-dependent dynamics and regulation of mitotic kinesins in Drosophila S2 cells. Mol Biol Cell. 2005;16:3896-907.

37. Byers B, Goetsch L. A highly ordered ring of membrane-associated filaments in budding yeast. J Cell Biol. 1976;69:717-21.

38. Pan F, Malmberg RL, Momany M. Analysis of septins across kingdoms reveals orthology and new motifs. BMC Evol Biol. 2007;7:103.

39. Boyce KJ, et al. An Ustilago maydis septin is required for filamentous growth in culture and for full symptom development on maize. Eukaryot Cell. 2005;4:2044-56.

40. Dagdas $Y F$, et al. Septin-mediated plant cell invasion by the rice blast fungus, Magnaporthe oryzae. Science (New York, NY). 2012;336:1590-5.
41. Iwase M, et al. Forchlorfenuron, a phenylurea cytokinin, disturbs septin organization in Saccharomyces cerevisiae. Genes Genet Syst. 2004;79:199-206.

42. Angelis $\mathrm{D}$, et al. In silico docking of forchlorfenuron (FCF) to septins suggests that FCF interferes with GTP binding. PLoS One. 2014;9:e96390.

43. Brambl R. Characteristics of developing mitochondrial genetic and respiratory functions in germinating fungal spores. Biochim Biophys Acta. 1975;396:175-86.

44. Taubitz A, et al. Role of respiration in the germination process of the pathogenic mold Aspergillus fumigatus. Curr Microbiol. 2007;54:354-60.

45. Pungartnik $C$, et al. Reactive oxygen species and autophagy play a role in survival and differentiation of the phytopathogen Moniliophthora perniciosa. Fungal Genet Biol. 2009;46:461-72.

46. Viaud $M$, et al. Cyclophilin $A$ and calcineurin functions investigated by gene inactivation, cyclosporin A inhibition and CDNA arrays approaches in the phytopathogenic fungus Botrytis cinerea. Mol Microbiol. 2003;50:1451-65.

47. Lin HC, et al. The fumagillin biosynthetic gene cluster in Aspergillus fumigatus encodes a cryptic terpene cyclase involved in the formation of beta-trans-bergamotene. J Am Chem Soc. 2013;135:4616-9.

48. Wiemann P, et al. Prototype of an intertwined secondary-metabolite supercluster. Proc Natl Acad Sci U S A. 2013;110:17065-70.

49. Schumann J, Hertweck C. Advances in cloning, functional analysis and heterologous expression of fungal polyketide synthase genes. J Biotechnol. 2006;124:690-703.

50. Butler MJ, Day AW. Fungal melanins: a review. Can J Microbiol. 1998:44:1115-36.

51. Eisenman HC, Casadevall A. Synthesis and assembly of fungal melanin. Appl Microbiol Biotechnol. 2012:93:931-40.

\section{Submit your next manuscript to BioMed Central and we will help you at every step:}

- We accept pre-submission inquiries

- Our selector tool helps you to find the most relevant journal

- We provide round the clock customer support

- Convenient online submission

- Thorough peer review

- Inclusion in PubMed and all major indexing services

- Maximum visibility for your research

Submit your manuscript at www.biomedcentral.com/submit
) Biomed Central 